\title{
Depletion Layers and Domain Walls in Semiconducting Ferroelectric Thin Films
}

\author{
Yu Xiao, ${ }^{1}$ Vivek B. Shenoy, ${ }^{2}$ and Kaushik Bhattacharya ${ }^{1, *}$ \\ ${ }^{1}$ Division of Engineering and Applied Science, California Institute of Technology, Pasadena, California 91125, USA \\ ${ }^{2}$ Division of Engineering, Brown University, Providence, Rhode Island 02912, USA
}

(Received 27 August 2005; published 9 December 2005)

\begin{abstract}
Commonly used ferroelectric perovskites are also wide-band-gap semiconductors. In such materials, the polarization and the space-charge distribution are intimately coupled, and this Letter studies them simultaneously with no a priori ansatz on either. In particular, we study the structure of domain walls and the depletion layers that form at the metal-ferroelectric interfaces. We find the coupling between polarization and space charges leads to the formation of charge double layers at the $90^{\circ}$ domain walls, which, like the depletion layers, are also decorated by defects like oxygen vacancies. In contrast, the $180^{\circ}$ domain walls do not interact with the defects or space charges. Implications of these results to domain switching and fatigue in ferroelectric devices are discussed.
\end{abstract}

Ferroelectric perovskites that are widely used as actuators, sensors, and memories have classically been modeled as insulators following the Devonshire-Ginsburg-Landau (DGL) theory [1]. These materials, however, are also wideband-gap semiconductors [2]. Consequently, one has bandbending and depletion layers at electrodes and charge layer formation at domain walls, which in turn affect the polarization distribution. These in turn contribute to fatigue and domain-wall pinning.

Recent efforts have sought to augment the DGL theory for these effects [3]. However, they assume a priori the depletion layer and space-charge distribution and calculate the resulting polarization distribution, or vice versa. There have also been $a b$ initio studies of the electronic band structure and atomistic studies of defects [4-6]. However, they provide limited information at the device scale due to enormous computational effort required to handle realistic length scales and geometries. This Letter provides a comprehensive presentation of a semiconducting ferroelectric at the device scale with no a priori assumptions on the polarization or space charge.

We focus on the [001]-polarized tetragonal phase of $\mathrm{BaTiO}_{3}$ to be specific, though many of our findings are generic. This well-studied material displays both $180^{\circ}$ and $90^{\circ}$ domain walls. Oxygen vacancies are a common defect and they act as donors $[6,7]$. We focus on the parallel-plate capacitor geometry (electrode-ferroelectric-electrode), which is the building block of many devices. Our results show the formation of depletion layers and the alteration of the polarization near electrodes, reveal some essential differences between $180^{\circ}$ and $90^{\circ}$ domain walls, show the redistribution of oxygen vacancies to electrodes and across $90^{\circ}$ domain walls during annealing, and provide a concrete mechanism for imprinting a $90^{\circ}$ domain wall. These results shed new light on various experimental observations.

The state of the semiconducting ferroelectric crystal is determined by the polarization density $\mathbf{p}$, the strain $\boldsymbol{\epsilon}$, the space-charge density $\rho$, and the defect density $N_{d}$, each of which we treat as field quantities. We assume here that all defects are donors motivated by oxygen vacancies in $\mathrm{BaTiO}_{3}$, but this can be readily modified to include acceptors. The space charge reflects the electrons donated by the defects, the electrons in the conduction band, the holes in the valence band, and depends on the electrostatic potential as follows [8]:

$$
\begin{aligned}
\rho\left(\phi, N_{d}\right)= & e z f(\phi) N_{d}-e N_{c} F_{1 / 2}\left(\frac{E_{\mathrm{Fm}}-E_{c}+e \phi}{K_{B} T}\right) \\
& +e N_{v} F_{1 / 2}\left(\frac{E_{v}-e \phi-E_{\mathrm{Fm}}}{K_{B} T}\right) ; \\
f(\phi)= & 1-\left[1+\frac{1}{g} \exp \left(\frac{E_{d}-e \phi-E_{\mathrm{Fm}}}{K_{B} T}\right)\right]^{-1} .
\end{aligned}
$$

$N_{c}\left(N_{v}\right)$ is the effective density of states in the conduction (valence) band, $E_{c}\left(E_{v}\right)$ the conduction (valence) band edge energy, $E_{d}$ the donor level, $E_{\mathrm{Fm}}$ the Fermi level of the semiconductor, $F_{1 / 2}$ the Dirac-Fermi integral, $T$ the absolute temperature, $z$ the donor valency, $f(\phi)$ the fraction of ionized donors, and $g$ the ground state degeneracy of the donor impurity level [9].

The density of defects can evolve through diffusion,

$$
\frac{\partial N_{d}}{\partial t}=\boldsymbol{\nabla} \cdot\left[\beta N_{d} \boldsymbol{\nabla}\left(\frac{\partial W_{d}}{\partial N_{d}}+e z f(\phi) \phi\right)\right],
$$

where the term in the inner parenthesis is the chemical potential, $\beta$ is the mobility, and $W_{d}$ is the contribution to the free energy due to defects which is assumed to be the usual free energy of mixing at small concentrations [10]. The polarization density, the space-charge density, and the electrostatic potential must be consistent with charge conservation (Gauss's or Maxwell's equation)

$$
\boldsymbol{\nabla} \cdot\left(-\epsilon_{0} \boldsymbol{\nabla} \phi+\mathbf{p}\right)=\rho\left(\phi, N_{d}\right),
$$

where $\epsilon_{0}$ is the permittivity of free space. The polarization evolves as in the time-dependant DGL theory: 


$$
\mu \frac{\partial \mathbf{p}}{\partial t}=\boldsymbol{\nabla} \cdot\left(\frac{\partial W}{\partial \nabla \mathbf{p}}\right)-\frac{\partial W}{\partial \mathbf{p}}-\boldsymbol{\nabla} \phi
$$

where $1 / \mu$ is the mobility and the energy density $W$ is

$$
W=\frac{a_{0}}{2}|\nabla \mathbf{p}|^{2}+W_{p}(\mathbf{p}, \varepsilon) .
$$

The first term is the domain-wall energy, and the second term is the classical Devonshire energy (with minima at $\mathbf{p}=p_{0}\langle 001\rangle$, where $p_{0}$ is saturation polarization), chosen to be as in Ref. [11] for $\mathrm{BaTiO}_{3}$. Finally, the strain $\boldsymbol{\epsilon}$ is decided by mechanical equilibrium,

$$
\boldsymbol{\nabla} \cdot \boldsymbol{\sigma}=0,
$$

where $\boldsymbol{\sigma}=\partial W / \partial \boldsymbol{\epsilon}$ is the Cauchy stress.

The Eqs. (2)-(4) and (6), along with (1) and (5) have to be solved subject to appropriate boundary and initial conditions. We do so in two dimensions for a $\mathrm{BaTiO}_{3}$ single crystal slab (whose pseudocubic axes are aligned with the slab) with shorted platinum electrodes on both faces using the finite element method. We assume that $\beta \ll 1 / \mu$, so that the time scale is too short for diffusion of defects but long enough for the formation of domains. We therefore fix $N_{d}$ and evolve $\mathbf{p}$ until equilibrium is achieved. Two representative results are shown in Fig. 1.

Figures 1(a) and 1(b) show the results of a simulation with a $180^{\circ}$ domain wall in the computational domain

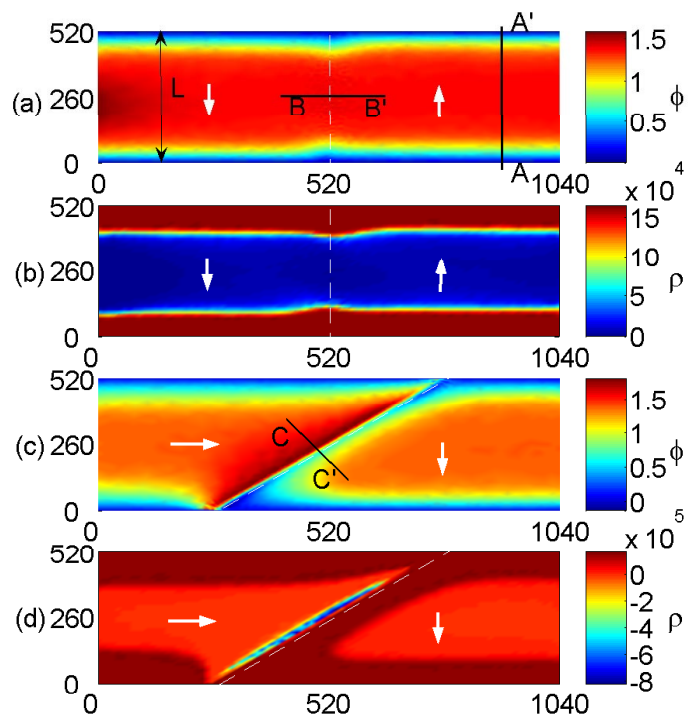

FIG. 1 (color online). (a), (c) Electric potential (V) and (b), (d) space-charge densities $\left(\mathrm{Cm}^{-3}\right)$ of $\mathrm{a} \mathrm{BaTiO}_{3}$ slab $(1040 \mathrm{~nm} \times$ $520 \mathrm{~nm}$ ) with shorted Pt electrodes. In cases (a), (b) a $180^{\circ}$ domain wall and in (c), (d) a $90^{\circ}$ domain wall is present in the slab. The location of domain walls is marked by white dashed lines, and the polarization directions are indicated by white arrows. For the material parameters for $\mathrm{BaTiO}_{3}$ with oxygen vacancies see Refs. [9,11]. The density of oxygen vacancies is $N_{d}=10^{24} \mathrm{~m}^{-3}$ and $a_{0}=10^{-7} \mathrm{~V} \mathrm{~m}^{3} \mathrm{C}^{-1}$. The last is high, corresponding to a domain-wall width of $50 \mathrm{~nm}$, but is convenient for visualization of the qualitative features. while Figs. 1(c) and 1(d) show the results with a $90^{\circ}$ domain wall. The results show the formation of depletion layers (of approximately $100 \mathrm{~nm}$ thickness) near the electrodes accompanied by a voltage increase of $1.4 \mathrm{~V}$ across the depletion layers. Further, we notice that there is an accumulation of charges near the $90^{\circ}$ domain wall and a potential drop across this wall. These features are absent at the $180^{\circ}$ domain wall. We examine each of these aspects in detail below.

The depletion layer plays an extremely important role in determining the charge injection from the electrode to the ferroelectric device, and consequently the leakage fatigue behavior of a ferroelectric device [2,12,13]. Further, Dawber et al. [13] find that extrapolating the $1 / C(C$ is the capacitance) vs thickness measurements in lead-zirconate-titanate (PZT) films to zero thickness gives a nonzero intercept providing evidence for its presence. To gain further insight, we study the profiles away from domain walls and transverse to the slab, along the section marked $A-A^{\prime}$ in Fig. 1. The results are shown in Fig. 2 for the cases where the defect diffusion is small $[\beta \ll 1 / \mu$, shown by solid curves (blue online)]. A small calculation shows that the build-in potential $\phi_{\text {bi }}$ of $1.4 \mathrm{~V}$ is approximately equal to the difference between the Fermi levels of the film and the electrode [14]. Since it acts as a barrier against charge injection, metal electrodes with higher work function or smaller Fermi level would be less susceptible to fatigue. This is consistent with observations [2]. Further, we can show that the width of the depletion layer is given by $d \approx$ $\sqrt{\left(2 \varepsilon_{r} \varepsilon_{0} \phi_{b i}\right) /\left(e z N_{d}\right)}$, where $\epsilon_{r}=\left[\left.\left(\partial^{2} W / \partial p^{2}\right)\right|_{p_{0}} \epsilon_{0}\right]^{-1}$ can be viewed as the effective relative dielectric constant. Since smaller depletion layers make it more susceptible to charge injection through tunneling, it follows that failure becomes worse with increasing doping density and better

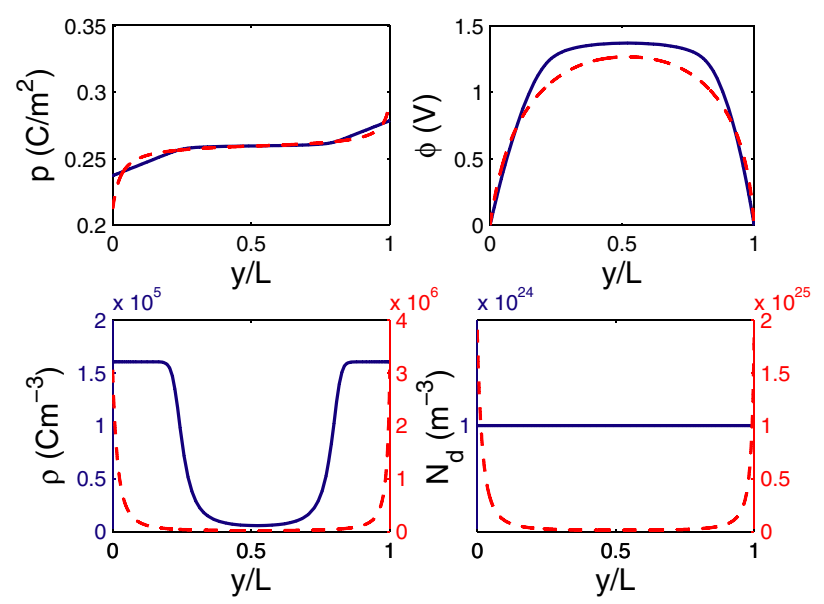

FIG. 2 (color online). The polarization, electric potential, space-charge density, and defect density along the line $A-A^{\prime}$ of the $\mathrm{BaTiO}_{3}$ slab in Fig. 1. Solid (blue online) lines: without diffusion of defects; dashed (red online) lines: with diffusion of defects. 
with large effective dielectric modulus. We present the detailed influence of the doping level on depletion layer elsewhere, including a finding of a "head-to-head" domain wall with an interior depletion layer in films above a critical doping level.

The large electric field in the depletion layers also drives the diffusion of defects including oxygen vacancies, especially during annealing at high temperatures, when $\beta \approx$ $1 / \mu$. To understand this, we solve diffusion equation (2) along with (3) and (4) until steady state is reached in both $N_{d}$ and $\mathbf{p}$. The results given by dashed (red online) curves in Fig. 2 indicate that the oxygen vacancies are almost fully depleted from the interior and tend to accumulate near the electrodes. The increased donor composition near the electrodes may accelerate failure for the reasons described earlier. Finally, this calculation shows that oxygen vacancies can be rearranged even when their total number is conserved. Thus the experiments of Nuffer et al. [15] that measure the amount of oxygen released from a specimen as a means of evaluating the role of oxygen vacancies in fatigue do not capture this mechanism of fatigue.

We now consider the polarization profiles transverse to isolated domain walls, i.e., along the sections $B$ - $B^{\prime}$ and $C-C^{\prime}$ in Fig. 1. In order to understand the observed trends, it is useful to rescale the polarization by the saturation polarization $p_{0}$, the energy density $W_{p}$ by the depth (difference between maxima and minima) of its wells $E_{b}$, the electrostatic potential by $\phi_{0}=\sqrt{a_{0} E_{b}}$ and the lengths by the domain-wall width, and $l_{0}=p_{0} \sqrt{a_{0} / E_{b}}$, where $a_{0}$ is the coefficient of the gradient term in (5) [16]. We first consider the simplest case where no defects are present in the crystal. The governing equations [17] then reduce to

$$
\begin{gathered}
\frac{d^{2} p_{r}^{\prime}}{d r^{\prime 2}}-\frac{\partial W_{p}^{\prime}}{\partial p_{r}^{\prime}}-\frac{d \phi^{\prime}}{d r^{\prime}}=0, \quad \frac{d^{2} p_{s}^{\prime}}{d r^{\prime 2}}-\frac{\partial W_{p}^{\prime}}{\partial p_{s}^{\prime}}=0, \\
-\epsilon^{\prime} \frac{d^{2} \phi^{\prime}}{d r^{\prime 2}}+\frac{d p_{r}^{\prime}}{d r^{\prime}}=0,
\end{gathered}
$$

where primes denote scaled variables, $\epsilon^{\prime}=\epsilon_{0} E_{b} / p_{0}^{2}$, and $r^{\prime}$ and $s^{\prime}$ are coordinates normal and parallel to the domain wall, respectively [18]. These equations differ from those used in the classical studies of domain walls [19], where it is assumed a priori that the normal component of the polarization $p_{r}^{\prime}$ and the electrostatic potential $\phi^{\prime}$ are constant, and therefore the first and third equations of (7) are omitted. Finally, to solve these equations in closed form, we choose

$$
W_{p}^{\prime}=\frac{a^{\prime}}{2}\left(p_{x}^{\prime 2}+p_{y}^{\prime 2}\right)+\frac{b^{\prime}}{2} p_{x}^{\prime 2} p_{y}^{\prime 2}+\frac{c^{\prime}}{4}\left(p_{x}^{\prime 4}+p_{y}^{\prime 4}\right)
$$

where $a^{\prime}=-4, c^{\prime}=-a^{\prime}=4$, and $b^{\prime}=20$. We have verified that the results obtained with this choice hold qualitatively for any generic form of $W_{p}^{\prime}$.

For a $180^{\circ}$ domain wall, the classical kink solution [20], $p_{r}^{\prime}=0, p_{s}^{\prime}=\tanh \left(\sqrt{2} r^{\prime}\right)$, and $\phi^{\prime}=$ const also solves the more general system of Eq. (7). The situation is very different for a $90^{\circ}$ domain wall. It is easy to verify that the classical kink solution [20] with $p_{r}^{\prime} \equiv \frac{1}{\sqrt{2}}$ and $\phi^{\prime} \equiv 0$ does not solve (7). While we are unable to obtain an exact solution to (7) in closed form, we are able to obtain a perturbative solution using the fact that $\epsilon^{\prime} \approx 10^{-4}$ for $\mathrm{BaTiO}_{3}$ :

$$
\begin{gathered}
p_{r}^{\prime}=\frac{1}{\sqrt{2}}\left[1-2 \epsilon^{\prime} \operatorname{sech}\left(\sqrt{3} r^{\prime}\right)\right], \quad p_{s}^{\prime}=-\frac{1}{\sqrt{2}} \tanh \left(\sqrt{3} r^{\prime}\right), \\
\phi^{\prime}=-\frac{\sqrt{6}}{3}\left[\tanh \left(\sqrt{3} r^{\prime}\right)+1\right] .
\end{gathered}
$$

This approximates well the exact numerical solution shown in the solid black lines in Fig. 3. The key feature of this solution is that the transverse component of the polarization $\left(p_{r}^{\prime}\right)$ shows a dip at the domain wall, and consequently one has a drop in voltage of $0.115 \mathrm{~V}$ across the $90^{\circ}$ domain wall. It is interesting to note that Mayer and Vanderbilt [4] obtained a comparable voltage drop $(0.15-0.2 \mathrm{~V})$ across the $90^{\circ}$ domain wall in $\mathrm{PbTiO}_{3}$ using ab initio methods; in their paper [4], they call this result "surprising" as it is not anticipated on the basis of the classical description of domain walls [20]. Here, we have shown that the voltage drop is a natural outcome of solving the set of coupled equations in (7). We also point out that the width of the domain walls for the two types of domain boundaries $(\sim 4 \mathrm{~nm})$ and the ratio of the formation energy of the $180^{\circ}$ wall to the $90^{\circ}$ wall (3.27) also compare favorably with their results (width of $5 \mathrm{~nm}$ and energy ratio of 4) of ab intio calculations [4].

The variation in the electrostatic potential across the $90^{\circ}$ walls provides a driving force for the rearrangement of
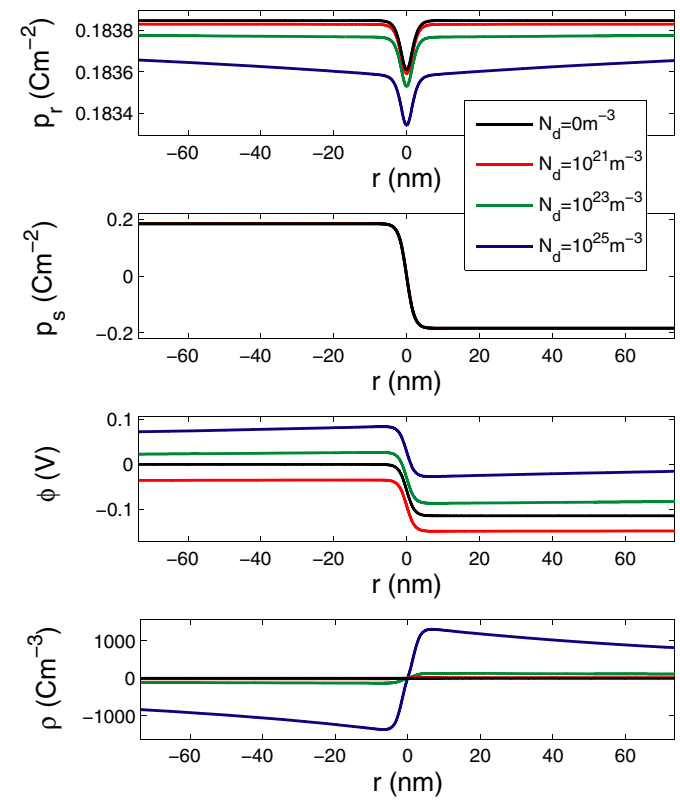

FIG. 3 (color online). Structure of an isolated $90^{\circ}$ domain wall in $\mathrm{BaTiO}_{3}$ [16] plotted as a function of the coordinate transverse to the wall $(r)$ for different density of donors $\left(N_{d}\right)$. 

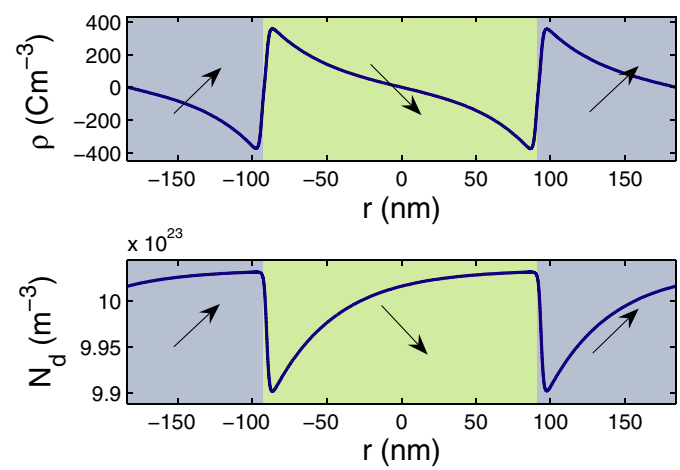

FIG. 4 (color online). Distribution of space charge and donors (average density $N_{d}=10^{24} \mathrm{~m}^{-3}$ ) for a periodic array of $90^{\circ}$ domain walls in $\mathrm{BaTiO}_{3}$ [16]. In this calculation, the defects are allowed to diffuse and achieve a steady state. Notice the formation of charge double layers and asymmetric redistribution of defects near the domain walls. The shading represents different domains with polarization direction indicated by arrows.

space charges and defects [refer to Fig. 1(d)]. In distinct contrast, there is no interaction between the $180^{\circ}$ walls and space charges. To explore this further, we have plotted the variation of field quantities across the $90^{\circ}$ domain walls as a function of the doping density in Fig. 3, for the case where the defect diffusion is negligible. With increasing density, a larger space-charge density builds on the sides of the domain wall, while the polarization and the electrostatic potential retain the general features as in the undoped case. If diffusion of defects is allowed, we expect them to decorate the domain walls as in the case of the depletion layer. This is indeed what is observed for a periodic array of domain walls in Fig. 4, where in steady state, the dopants segregate along a domain wall in an asymmetric manner creating a defect dipole. Recent simultaneous AFM-PFM measurements of emerging $90^{\circ}$ domain walls in $\mathrm{BaTiO}_{3}$ show that the physical width is significantly smaller (over 7 times) than the piezoelectric width consistent with longrange electrostatic interactions [21]. Further, the decoration of the domain wall with defects is consistent with experimental observations of Shilo et al. [22] and provides a mechanism for the domain wall to have a memory of its location during annealing. To understand this mechanism, note that the domains that disappear on the application of an electric field will not, in general, reappear at their old locations when the electric field is switched off. However, the inhomogeneous charge distribution allows the walls to have a memory of their location (by providing favorable nucleation sites) even after the field is switched off. Thus, it provides a mechanism for imprinting the domain wall and for the large electrostriction through aging recently observed by Ren [23]. Finally, the presence of these charge and defect layers at the $90^{\circ}$ domain walls means that such walls promote electrical failure by providing a high conductivity pathway from electrode to electrode.
We gratefully acknowledge the discussions with Jim Scott and Wei Zhang, and the financial support of the US Army Research Office through the MURI Grant No. DAAD 19-01-1-0517 and National Science Foundation through Grants No. CMS-0093714 and No. CMS0210095.

*Electronic address: bhatta@its.caltech.edu

[1] A. F. Devonshire, Adv. Phys. 3, 85 (1954).

[2] J. F. Scott, Ferroelectric Memories (Springer, New York, 2000).

[3] A. Tagantsev, C. Pawlaczyk, K. Brooks, M. Landivar, E. Colla, and N. Setter, Integr. Ferroelectr. 6, 309 (1995); A. Bratkovsky and A. Levanyuk, Phys. Rev. B 61, 15042 (2000); Y. Watanabe, Appl. Surf. Sci. 132, 610 (1998); Y. Watanabe, Phys. Rev. B 57, 789 (1998).

[4] B. Meyer and D. Vanderbilt, Phys. Rev. B 65, 104111 (2002).

[5] M. Calleja, M.T. Dove, and E. K.H. Salje, J. Phys. Condens. Matter 15, 2301 (2003).

[6] Q. Zhang, Ph.D. thesis, California Inst. of Technology (2004)

[7] A.J. Moulson and J.M. Herbert, Electroceramics: Materials, Properties, Applications (Wiley, New York, 2003).

[8] S. M. Sze, Physics of Semiconductor Devices (Wiley, New York, 1981).

[9] We assume $z=1, g=2 ; E_{c}, E_{v}$, and $E_{d}$ (in eV) are chosen to be $-3.6,-6.6$, and -4.0 respectively, consistent with Refs. [2,6,7].

[10] D. A. Porter and K. E. Easterling, Phase Transformations in Metals and Alloys (Van Nostrand Reinhold, New York, 1981).

[11] W. Zhang and K. Bhattacharya, Acta Mater. 53, 185 (2005); Proceedings of SPIE Conference on Smart Materials, Vol. 5053 (SPIE-International Society for Optical Engineering, Bellingham, WA, 2003).

[12] B. Nagaraj, S. Aggarwal, and R. Ramesh, J. Appl. Phys. 90, 375 (2001).

[13] M. Dawber et al., Rev. Mod. Phys. 77, 1083 (2005).

[14] $\phi_{b i}=\left(E_{\mathrm{Fm}}^{B T}-E_{\mathrm{Fm}}^{P t}\right) / e \approx\left(E_{c}+E_{d}-2 E_{\mathrm{Fm}}^{P t}\right) / 2 e=1.5 \mathrm{~V}$.

[15] J. Nuffler et al., Appl. Phys. Lett. 79, 3675 (2001).

[16] For $\mathrm{BaTiO}_{3}$ at $T=300 \mathrm{~K}, p_{0}=0.26 \mathrm{Cm}^{-2}, E_{b}=5 \times$ $10^{6} \mathrm{Jm}^{-3}$ and $a_{0}=1 \times 10^{-9} \mathrm{~V} \mathrm{~m}^{3} \mathrm{C}^{-1}$.

[17] We ignore strain in this simple analysis, since numerical calculations (Fig. 1) show that its influence is secondary.

[18] For a tetragonal ferroelectric like $\mathrm{BaTiO}_{3}$ whose pseudocubic axes are oriented with the $x^{\prime}, y^{\prime}$ axes, $r^{\prime}=x^{\prime}, s^{\prime}=y^{\prime}$ and $r^{\prime}=\left(x^{\prime}-y^{\prime}\right) / \sqrt{2}, s^{\prime}=\left(x^{\prime}+y^{\prime}\right) / \sqrt{2}$ for $180^{\circ}$ and $90^{\circ}$ domain walls, respectively.

[19] W. Cao and L. E. Cross, Phys. Rev. B 44, 5 (1991).

[20] F. Falk, Z. Phys. B 51, 177 (1983).

[21] C. Franck, G. Ravichandran, and K. Bhattacharya (to be published).

[22] D. Shilo, G. Ravichandran, and K. Bhattacharya, Nat. Mater. 3, 453 (2004).

[23] X. Ren, Nat. Mater. 3, 91 (2004). 